\title{
Analysis of Approaches to Anti-tuberculosis Compounds
}

\author{
Sara Motamen and Ronald J. Quinn*
}

Cite This: ACS Omega 2020, 5, 28529-28540

Read Online

ABSTRACT: Mycobacterium tuberculosis $(M t b)$ remains a deadly pathogen two decades after the announcement of tuberculosis (TB) as a global health emergency by the World Health Organization. Medicinal chemistry efforts to synthesize potential drugs to shorten TB treatments have not always been successful. Here, we analyze physiochemical properties of $39 \mathrm{~TB}$ drugs and 1271 synthetic compounds reported in 40 publications from 2006 to early 2020 . We also propose a new TB space of physiochemical properties that may provide more appropriate guidelines for design of anti-TB drugs.

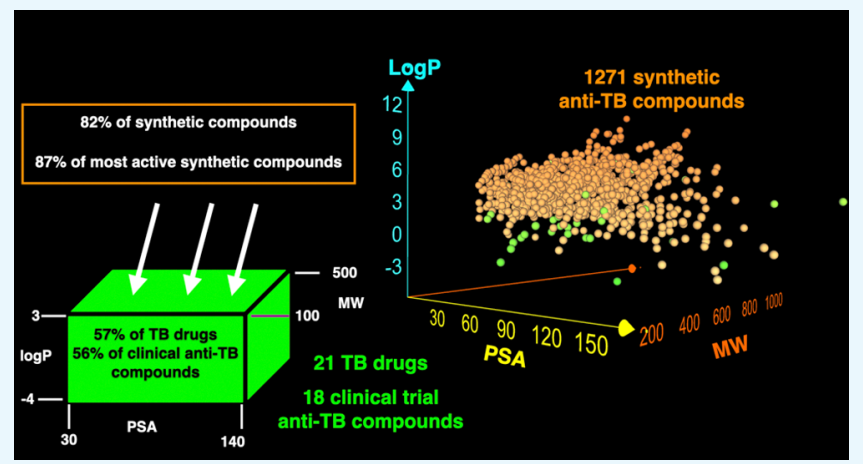

\section{INTRODUCTION}

Tuberculosis (TB), a communicable disease caused by Mycobacterium tuberculosis ( $M t b)$, is one of the top 10 leading causes of death worldwide and the leading cause of death from a single infectious disease (ranked above HIV/AIDS). ${ }^{1}$ It typically affects the lungs (pulmonary $\mathrm{TB}$ ) but can also have effects on other sites (extrapulmonary TB). This disease can be spread when people who are infected with pulmonary $\mathrm{TB}$ expel it to air, for instance, by coughing or sneezing. TB has remained a global health problem because $M t b$ adopts different strategies to survive in a variety of host lesions. The pathogen has become resistant to currently available drugs, and this is one of the main reasons for the failure to control the spread of TB.

The current initial treatment of TB involves taking four drugs (isoniazid 1, rifampicin 2, and pyrazinamide 3, and ethambutol 4) daily for two months, followed by four months of rifampicin and isoniazid in the continuation stage. This regimen is currently used for most cases of TB and has been successful in treatment of $80-90 \%$ of patients with drug-sensitive TB. However, due to the increasing number of multi-drug resistant TB (MDR-TB) and $\mathrm{HIV} / \mathrm{TB}$ co-infection cases, TB is still a leading cause of death worldwide. ${ }^{1}$ MDR-TB caused by $M t b$ bacilli that are resistant to at least rifampicin and isoniazid requires at least 20 months of treatment with drugs that are more toxic, poorly efficient, and poorly tolerated, with cure rates of only $60-70 \% .{ }^{1} \mathrm{~TB} / \mathrm{HIV}$ coinfection has more complications. In developing countries, $\mathrm{TB}$ is the main cause of death among HIV-infected people. As a result, interactions between anti-TB drugs and anti-retrovirals enhance the risk of adverse effects and make the treatment more complicated. TB treatment is challenging and requires early diagnosis, accurate and effective chemotherapy regimens, and drug-resistance screening. Development of shorter and simpler

drug regimens that are safe, suitable for joint TB/HIV treatment, and well tolerated is essential.

Here, we review synthetic anti-mycobacterium compounds reported in 40 publications from 2006 to 2020, selected to contain diverse chemical classes, and present an analysis of the drug-like properties of the reported compounds to inform better strategies for synthesis of new anti-TB compounds.

Assessing Physiochemical Properties and Druggability. In 1997, Lipinski proposed the "Rule of Five" (Ro5) as a result of the analysis of around $90 \%$ of orally active drug candidates that were in phase II clinical trials in order to understand which factors contributed to compound attrition in clinical development. ${ }^{2}$ Ro5 is a set of four simple physiochemical properties: hydrogen bond donor (HBD), hydrogen bond acceptor (HBA), molecular weight (MW), and logarithm of partition coefficient of a molecule between aqueous and lipophilic phases, usually octanol and water $(\log P)$. According to this rule, to be orally bioavailable, a candidate molecule should have the cut-off numbers of five or multiples of five in these four factors. Cell permeable compounds should have less than five HBDs ( $\mathrm{HBD} \leq 5)$, less than ten HBAs (HBA $\leq 10)$, a $\mathrm{MW} \leq 500$, and a $\log P \leq 5$. If two or more properties are violated in the Ro5 by a compound, there is a high probability of lack of bioavailability and oral activity. ${ }^{2}$ At the same time, there is no guarantee that the molecule is druggable if it passes the Ro5. The rule is used as a guide for better selection and design of

Received: July 1, 2020

Accepted: October 15, 2020

Published: October 27, 2020

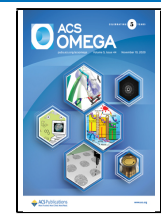


Table 1. Physiochemical Properties of 39 Approved TB Drugs and Candidates in Clinical Trials

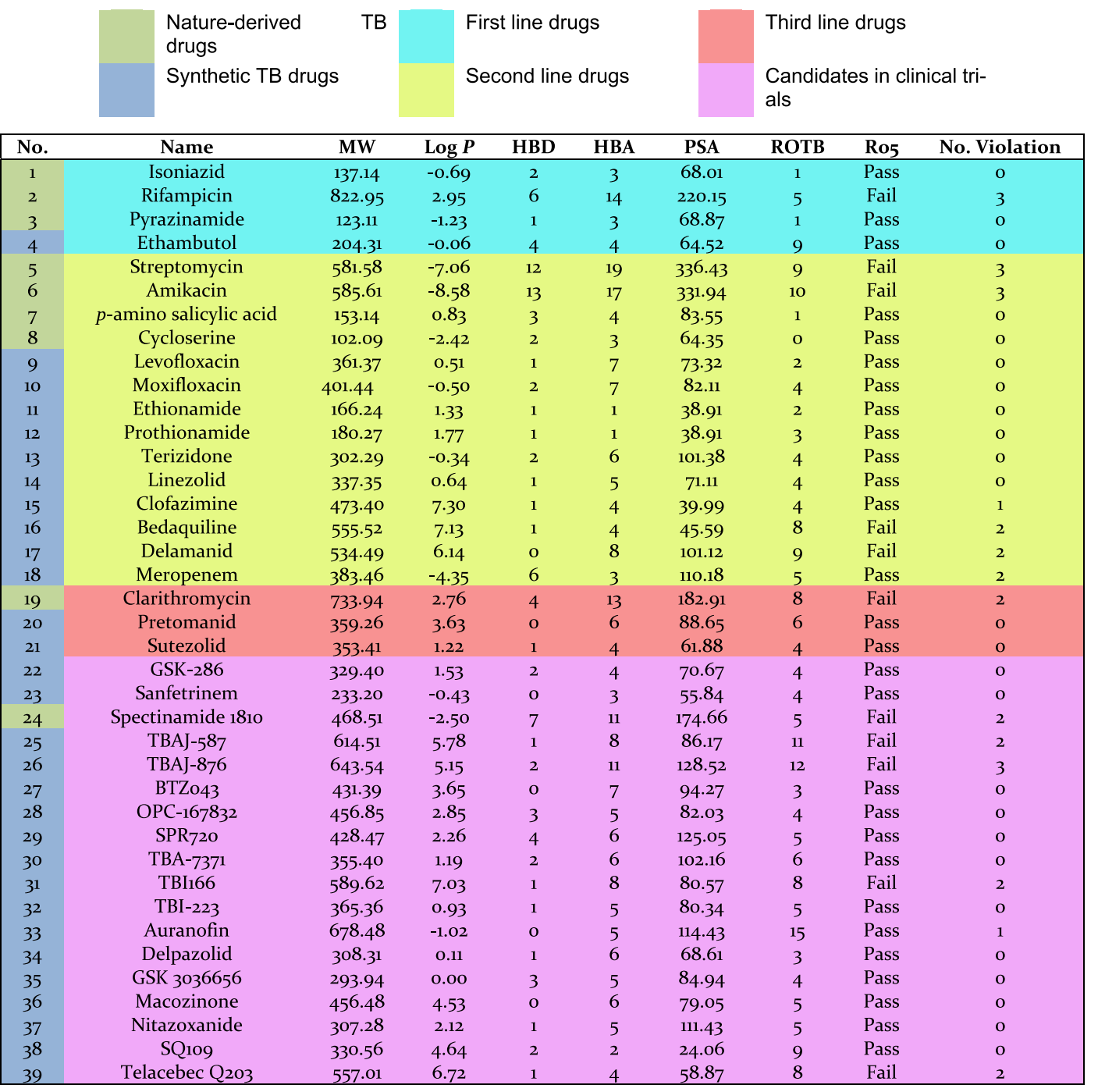
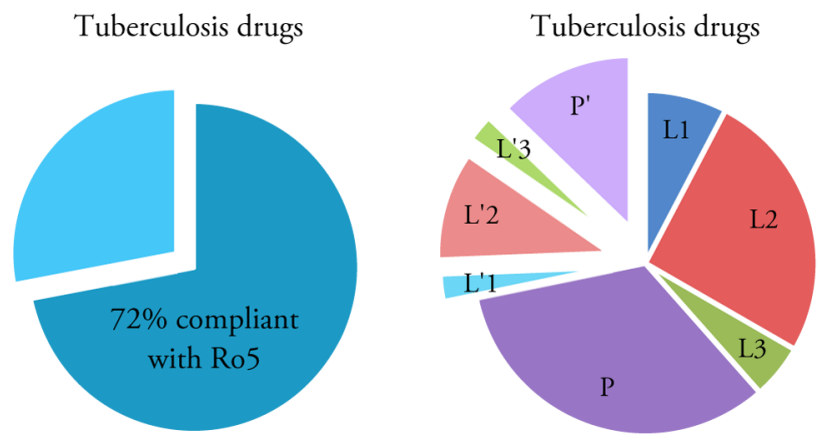

Synthetic compounds (20062020)

Figure 1. Pie chart presentation of the percentage of the TB drugs (left and middle) and analyzed anti-TB synthetic compounds (right) compliant with Lipinski's Ro5. L1, L2, L3, P: first, second, third line, and pipeline TB drugs compliant with Ro5. L'1, L'2, L'3, $\mathrm{P}^{\prime}$ : first, second, third line, and pipeline TB drugs not compliant with Ro5.

compounds to reduce attrition in clinical development due to unsatisfactory pharmacokinetics, and it is not an absolute set of strict guidelines. ${ }^{3}$

Lipinski's rule is sometimes misleading. For instance, some undesirable compounds can pass the Ro5 and, therefore, be considered druggable, whereas more appropriate compounds can fail due to the violation of one or more cut-offs. Subsequently, Hopkins and co-workers proposed "quantitative estimate of drug-likeness" (QED) which is a measure of druglikeness based on the concept of desirability. ${ }^{4}$ QED is an integrated function of eight desirability functions which are calculated for each physiochemical property including MW, log $P$, HBD, HBA, polar surface area (PSA), rotatable bonds (ROTBs), aromatic ring count (RNG), and number of alerts. One of the useful properties to predict oral bioavailability and activity is rotatable bond count. Each two ROTBs decrease 
a

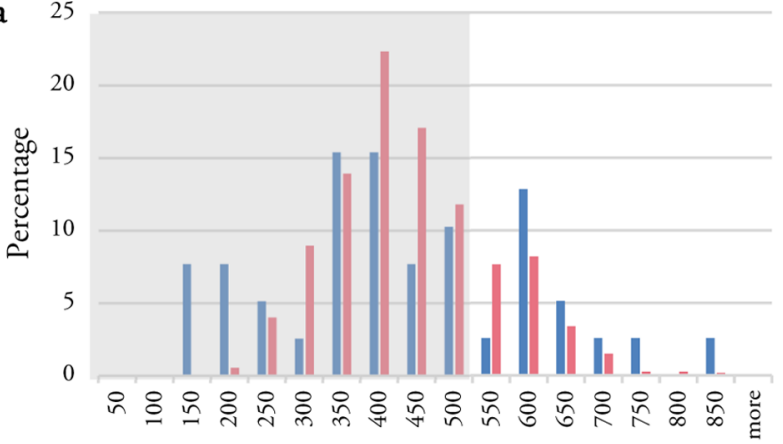

MW
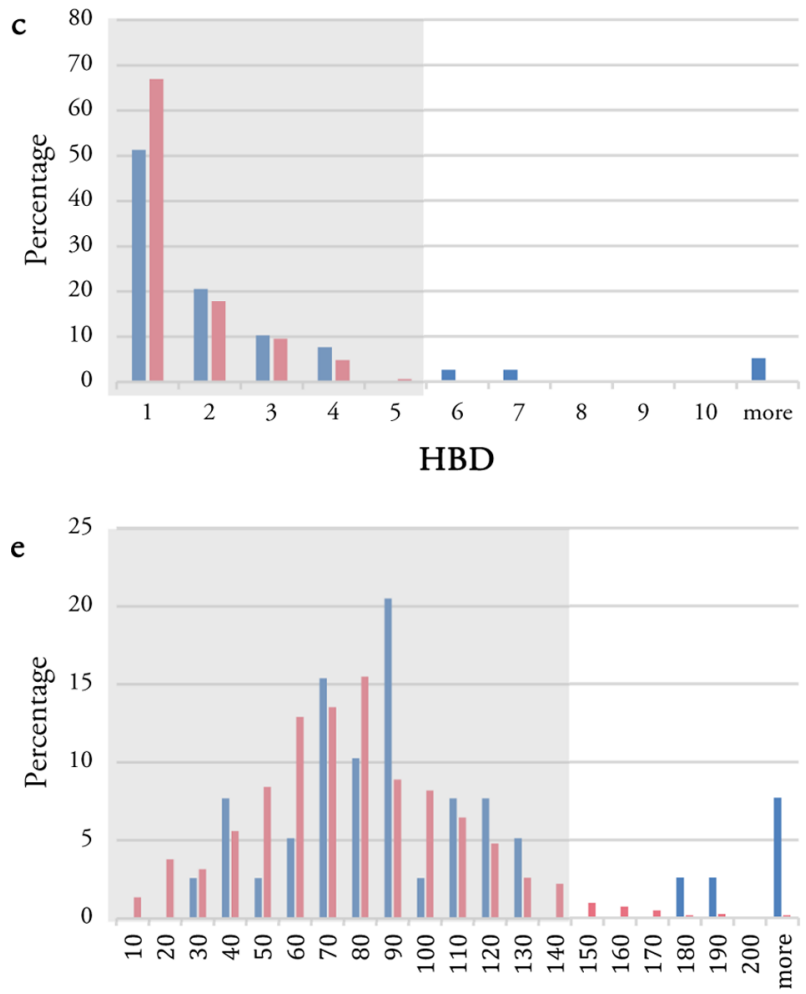

PSA
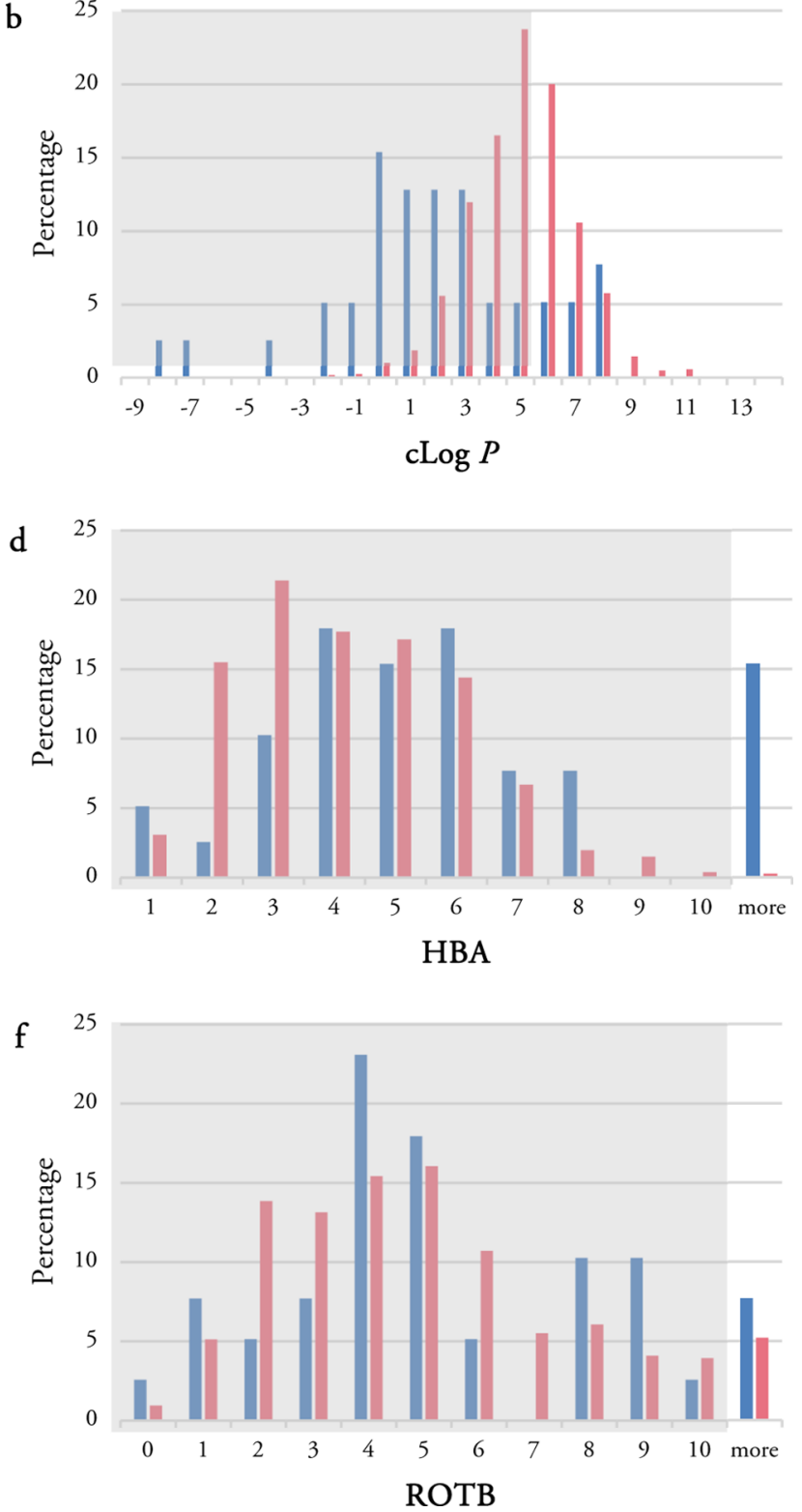

Figure 2. Physiochemical property histogram of TB drugs (blue) and anti-TB synthetic compounds (pink): (a) MW, (b) clog P, (c) HBDs, (d) HBAs, (e) PSA, and (f) ROTBs. The gray area shows preferred regions for compliance with Lipinski Ro5 or QED spaces.

ligand affinity by $0.5 \mathrm{kcal}$ on average. ${ }^{5}$ If both a rigid and a flexible ligand bind to a protein with the same pattern of interaction (based on hydrogen and hydrophobic interactions), the rigid ligand will have much stronger binding due to lower entropic losses. ${ }^{6}$ A good orally bioavailable drug usually has 10 or fewer ROTBs and polar surface equal to or less than $140 \AA$ as well as following Lipinski's Ro5. ${ }^{7}$ Although QED provides a richer and more reliable concept than Ro5, it is also not the final word in understanding drug-likeness features. In 1999, leadlikeness space was also proposed by Oprea in the following parameters: $\mathrm{MW} \leq 350$ and $\log P \geq 3$.

Herein, the four individual properties $\mathrm{MW}, \log P, \mathrm{HBD}$, and HBA as well as PSA and ROTB were analyzed for 39 TB drugs (approved and candidates in clinical trials) and 1271 synthetic anti-tubercular compounds reported in 40 publications between 2006 to early $2020 .^{9-48}$ Instant JChem 17.29.0 [Instant JChem 17.29.0, 2017 ChemAxon Ltd. (www.chemaxon.com)] was used for calculation of physiochemical properties. ${ }^{49}$

\section{RESULTS AND DISCUSSION}

Physiochemical Properties of TB Drugs. The calculated physiochemical properties of approved TB drugs and candidates in clinical trials are presented in Table $1 .^{50}$ It is also noted that anti-TB oxazolidinone posizolid (AZD5847) which completed a phase II clinical trial showed similar potential activities to other anti-TB agents listed; however, it is excluded from this analysis as its trial is discontinued. The percentage of TB drugs and synthetic anti-tubercular compounds compliant with Lipinski's rule is shown in Figure 1. While almost $72 \%$ of TB drugs (28 drugs) follow all Ro5 parameters or have just one violation, $28 \%$ of the drugs ( 11 drugs) have two or more violations. These values were 77 and $23 \%$, respectively, in our previous study published in $2014 .{ }^{51}$ Two of the drugs which violate Lipinski's rule are intravenous/injectable drugs (streptomycin 5 and amikacin 6). Four are orally bioavailable drugs (rifampicin 2, bedaquiline 16, delamanid 17, and clarithromycin 19) and five 

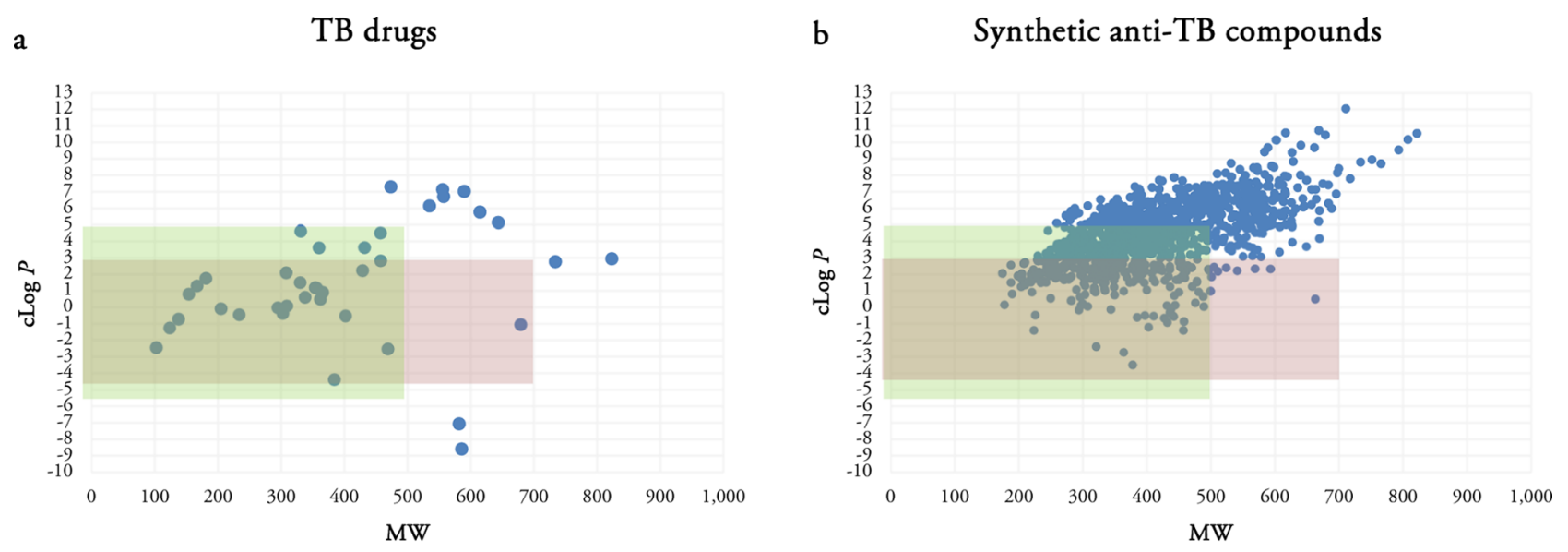

c Most active synthetic compounds

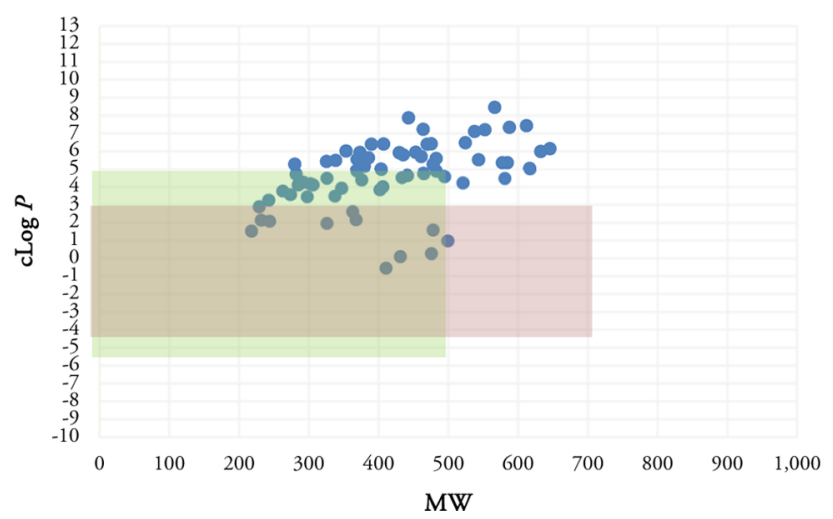

Figure 3. Scatterplots of TB drugs (a), all synthetic compounds (b), and the most active synthetic anti-TB compounds (c) using MW and clog $P$ as two variables. Green rectangle determines the Lipinski space and pink rectangle represents our putative TB space.

are in clinical trials (spectinamide 181024 , TBAJ-587 25, TBAJ876 26, and telacebec Q203 39).

The histogram for MW, calculated $\log P(\operatorname{cog} P), \mathrm{HBDs}$ and HBAs as well as PSA and ROTBs for TB drugs (blue bars) and the analyzed synthetic compounds (pink bars) are shown in Figure 2. The combined histograms for the most active compounds among the analyzed synthetic compounds can also be found in Supporting Information (Figure S1). About $49 \%$ of TB drugs have MWs of 300-500 Da and are all synthetic compounds either having novel structures such as TBI-223 32, delpazolid 34, and SQ109 38 or semisynthetic compounds derived from natural products such as spectinamide 181024 . About $28 \%$ of the TB drugs have the MWs of more than $500 \mathrm{Da}$. The lowest and highest MWs belong to nature-derived TB drugs. Cycloserine 8, pyrazinamide 3 , isoniazid 1 , and $p$ aminosalicylic acid 7 possess the lowest MWs of 100-150 Da, while the highest MWs of more than $700 \mathrm{Da}$ occur in rifampicin 2 and clarithromycin 19.

The distribution of $\operatorname{clog} P$ shows a wide range (Figure $2 \mathrm{~b}$ ). About $54 \%$ of TB drugs possess clog $P$ values in the range $-1 \leq$ $\operatorname{clog} P \leq 3$. About $18 \%$ have $\operatorname{cog} P$ of more than 5 , these include clofazimine 15, bedaquiline 16, delamanid 17, TBAJ-587 25, TBAJ-876 26, TBI166 31, and telacebec Q203 39. The most polar TB drug is the natural product streptomycin $\mathbf{5}$ with a $\operatorname{clog} P$ of about -7 . Overall, synthetic drugs have higher clog $P$ values than nature-derived TB drugs.

The distribution of HBDs shows a steady decrease starting from a maximum at 1 , while a wide range of variability is observed in the distribution histogram of HBAs. About $10 \%$ of the TB drugs violate the HBD cut-off values, while this number is slightly higher for HBA with about $15 \%$ violation. Rifampicin 2 , streptomycin 5, amikacin 6, and spectinamide 181024 are four TB drugs violating Lipinski's rule for both HBD and HBA values.

Two more physiochemical properties which have been analyzed are PSA and ROTBs. Violation of the desirable values for PSA and ROTB occur in about 13 and $8 \%$ of TB drugs, respectively. All TB drugs having PSAs of more than $140 \AA$ are nature-derived drugs. All three TB drugs having more than 10 ROTBs are synthetic drugs currently in clinical trials: auranofin 33, TBAJ-587 25, and TBAJ-876 26.

TB drugs have molecular exceptions to the Ro5. In the previous studies by O'Shea and Moser, it has been found that antibacterial compounds, especially for those active against Gram-negative bacteria, have higher average MWs and polarity in comparison to non-antibacterial compounds. ${ }^{52,53}$ This may be due to different cell wall architecture in Gram-positive and -negative bacteria which require different compound properties to be able to penetrate these two types of bacteria. ${ }^{52}$ The marketed TB drugs are widely distributed within the physiochemical space.

Physiochemical Properties of Synthetic Anti-mycobacterium Compounds Reported in 40 Publications from 2006 to 2020. A data set of 1271 synthetic anti-TB compounds reported in 40 publications from 2006 to 2020, selected to contain diverse chemical classes, was compiled to 
a

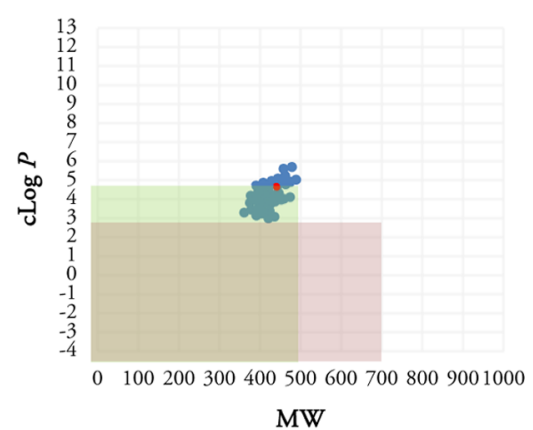

d

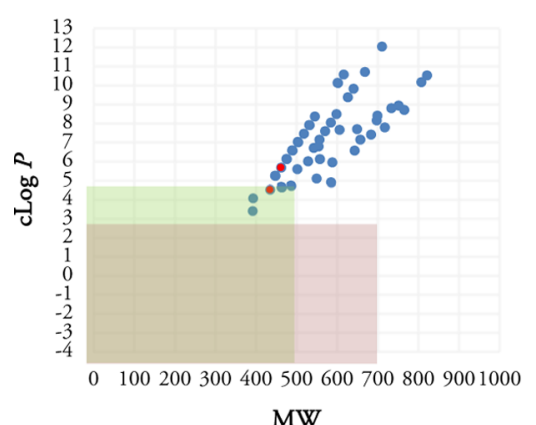

b

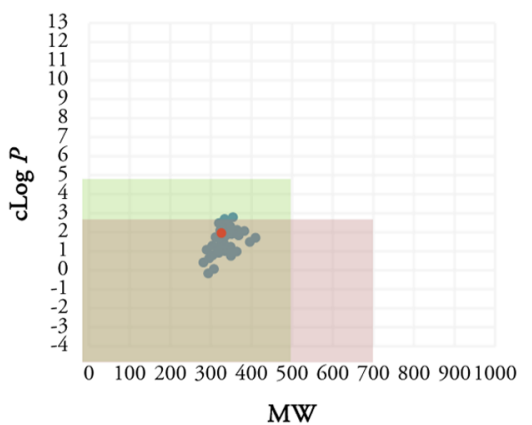

Publication 9

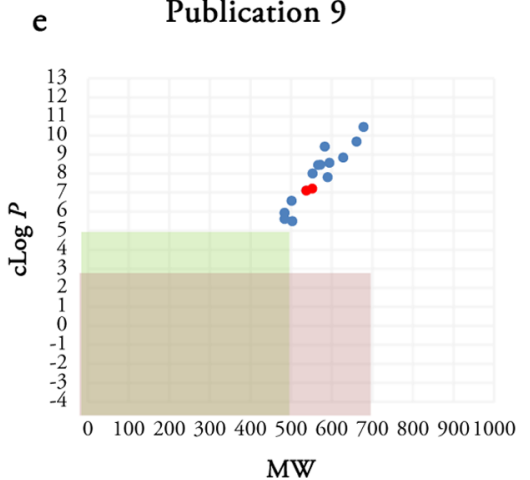

C

Publication 16

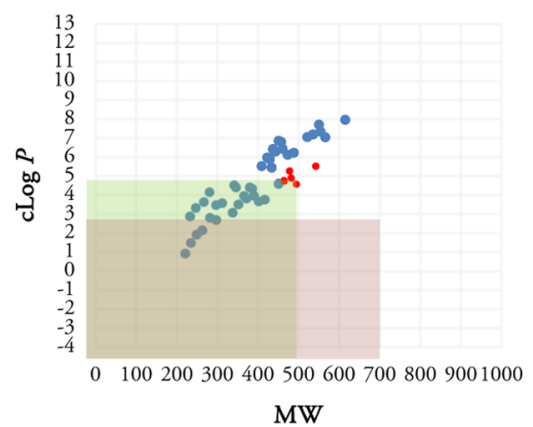

f Publication 4

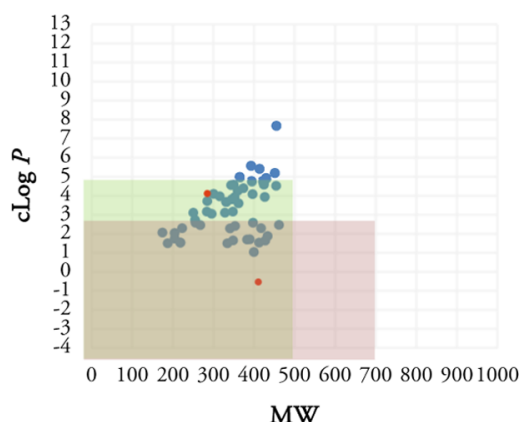

Figure 4. Scatterplots of the selected publications using MW and clog $P$ as two variables. Publication 5 (a), publication 28 (b), publication 16 (c), publication 19 (d), publication 9 (e), and publication 4 (f) are selected. The most active compounds in each series are shown as red dots. Green rectangle determines the Lipinski space, and pink rectangle represents our putative TB space.

analyze the strategies used in the design of these synthesized compounds. The majority of publications reported $15-50$ compounds. Only two publications reported less than 10 compounds and only one publication reported more than 50 compounds (115 compounds).

Histograms of the calculated physiochemical properties are depicted in Figure 2. Almost $83 \%$ of the synthetic compounds follow Lipinski's Ro5, and the majority of the remainder have violations in either $\mathrm{MW}, \operatorname{clog} P$, or both.

Unlike the wide variable distribution of TB drugs in the MW histogram, the synthetic compounds show a Gaussian distribution with a maxima at 350-400 Da. This distribution is similar to the distribution of anti-TB natural products analyzed in our previous studies. ${ }^{51,54}$ However, the percentage of natural products with MWs of more than $700 \mathrm{Da}$ was much higher than for the synthetic compounds. There is no synthetic compound with a MW of more than $1000 \mathrm{Da}$, while about $4 \%$ of the analyzed natural products were located in this region. About $21 \%$ of synthetic compounds violate Lipinski's rule with MWs of more than $500 \mathrm{Da}$. All 68 most active compounds reported in the analyzed 40 publications show a clog $P$ value between 200 and $650 \mathrm{Da}$ with about $80 \%$ following Lipinski's rule cut-off for MW (Figure S1).

The histogram of $\operatorname{clog} P$ shows the biggest variance between TB drugs and the synthetic compounds. The histogram of $\operatorname{clog} P$ for the synthetic compounds shows a Gaussian distribution as well as a maxima at 4-5; however, the synthetic drugs are shifted to a higher $\operatorname{cog} P$ values compared to TB drugs. This shift has also been observed in our previous studies for antimycobacterium natural products compared to all natural products. ${ }^{51}$ The histogram of $\operatorname{clog} P$ for TB drugs and anti- mycobacterial natural products depicted a bimodal pattern in our previous studies; however, this is not observed in the analysis of synthetic anti-mycobacterium compounds using the new updated TB drugs.

In contrast to $\mathrm{TB}$ drugs where about one-third have negative values of $\log P$, only $1.5 \%$ of synthetic compounds show negative clog $P$ values. The TB drugs distribution has the majority of compounds (54\%) with a $\operatorname{cog} P$ value between -1 and 3 , while $74 \%$ of synthetic compounds are observed in the range of the clog $P 3$ to $6.39 \%$ of the synthetic compounds violate Lipinski cut-off, whereas this is much smaller in TB drugs with $18 \%$ violation. The $\operatorname{cog} P$ histogram for the most active compounds reveals Gaussian distribution as well with a peak at 5-6. Out of 68 most active synthetic compounds, almost half (51\%) possess clog $P$ values between 4 and 6 . Only one compound shows a minus value $(-0.5)$ for $\operatorname{clog} P$, and none of them has $\operatorname{cog} P$ of more than 9.

The distribution of HBDs for the synthetic compounds is similar to that of TB drugs, showing a maximum at 1 followed by a steady decrease. The histogram of HBAs for the synthetic compounds reveals a wide range of variability similar to that of TB drugs. The noticeable difference is the percentage of violation from Lipinski cut-off between TB drugs and synthetic compounds in both HBD and HBA. The percentage of TB drugs violating Ro5 are 10 and $15 \%$ in HBD and HBA, respectively; however, this percentage is much less in synthetic compounds with less than $1 \%$ violation in both HBD and HBA. The histograms of HBD and HBA for most active compounds are also very similar to the related histograms of all synthetic compounds. About $6 \%$ of the most active compounds show 
a

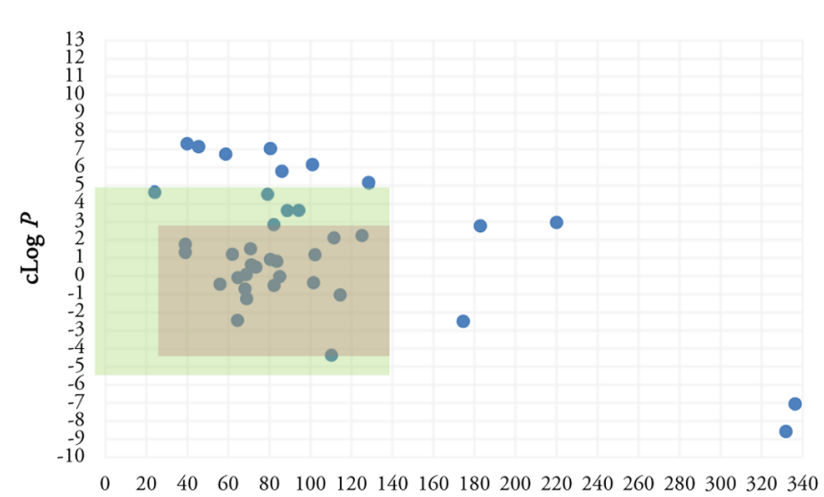

PSA

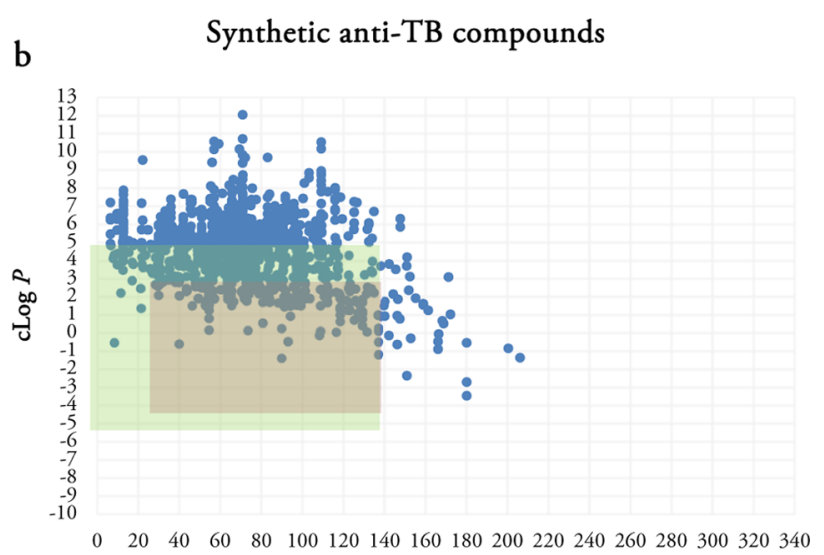

PSA

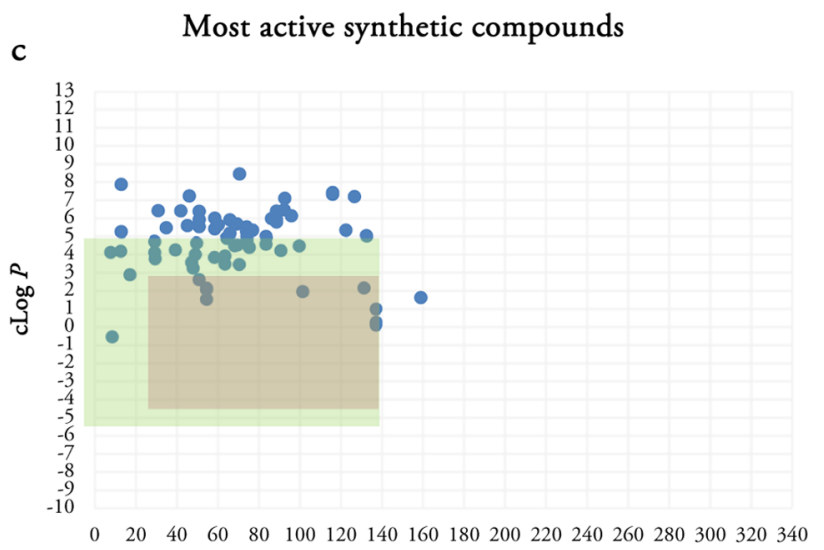

PSA

Figure 5. Scatterplots of the selected publications using $\operatorname{clog} P$ and PSA as two variables. Two physiochemical properties (clog $P$ and PSA) of TB drugs (a), synthetic anti-TB compounds (b), and the most active synthetic compounds (c) are evaluated. Green rectangle determines the QED space and pink rectangle represents our putative TB space.

HBD of more than 5, while all of them follow Lipinski's cut-off for HBA.

PSA shows Gaussian distribution with a peak at 70-80 ̊̊. Only $3 \%$ of the synthetic compounds have PSA of over $140 \AA$, while this percentage is higher in TB drugs with about $13 \%$ violation. The distribution of ROTBs is widely variable in synthetic compounds and TB drugs. The percentages of synthetic compounds and TB drugs which have more than 10 ROTBs are 5 and $8 \%$, respectively. The histograms of PSA and ROTB for most active compounds have also the same distribution as the related histograms of all synthetic compounds. The violations from Lipinski's rule in both cases are less than $5 \%$ in both PSA and ROTB.

Comparison of Anti-mycobacterial Synthetic Compounds Space Versus Current TB Drugs. We previously mentioned that antibacterial compounds have been reported to have higher average MWs and polarity in comparison to nonantibacterial compounds. ${ }^{52}$ The largest difference in our analysis was between the $\operatorname{cog} P$ values with many TB drugs having lower clog $P$ values. Herein, we investigated a putative TB space with $\mathrm{MW} \leq 700$ and $-4 \leq \operatorname{clog} P \leq 3$. Compounds with clog $P$ 's higher than 5 are often problematic from a safety perspective, and Figure $2 \mathrm{~b}$ shows that the majority of TB drugs have a clog $P$ $\leq 3$. Scatterplots of TB drugs, the synthetic compounds in each publication and in total, and the most active compound(s) in each publication using MW and $\operatorname{clog} P$ as two variables were analyzed (Figure 3).
There are 27 TB drugs in the Lipinski space and 23 in the putative TB space. Out of 1271 synthetic compounds, 719 are located in the Lipinski space and 266 are observed in the TB space. A large number of synthetic compounds possess large MWs and clog $P$. Out of 68 most active compounds in the analyzed publications, 56 compounds are compliant with Ro5 with none or just one violation. Half of the most active compounds (34 compounds) are located in the Lipinski space and 12 are observed in the TB space. While they are identified as the most active compounds in the respective series, it does not mean that they are active enough to be further evaluated or considered as the potent anti-TB compounds.

Scatterplots of some synthetic compounds in each publication using MW and clog $P$ as two variables are depicted in Figure 4. Remaining scatterplots are accessible in the Figure S2.

Different patterns have been observed in the analyzed scatterplots. In some publications such as publications $1,7,13$, $17,18,21,25,28,29,30,38$, and publication 5 shown in Figure $4 \mathrm{a}$, the synthetic compounds possess very similar MW and $\operatorname{clog} P$ values making clusters. The clusters are completely inside, partially inside, or completely outside the Lipinski or TB spaces in different publications. For example, publication 28 reported synthesis of anti-TB compounds making a cluster located in both Lipinski and putative TB spaces (Figure 4b).

Some scatterplots reveal a rising pattern of both MW and clog $P$ values among the reported synthetic compounds. Publications $3,6,9,16,19,22,24,26,27,32$, and 33 show this feature. These 
Publication 5

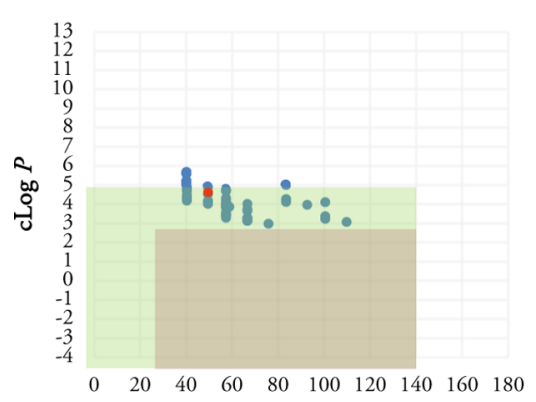

PSA

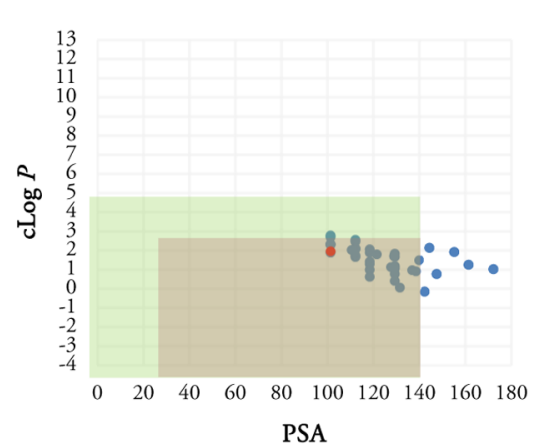

Publication 9

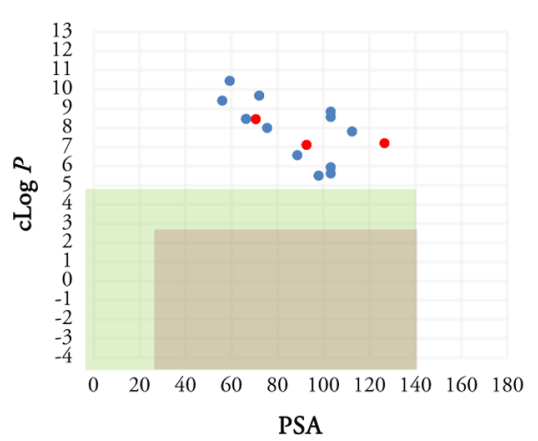

Publication 16

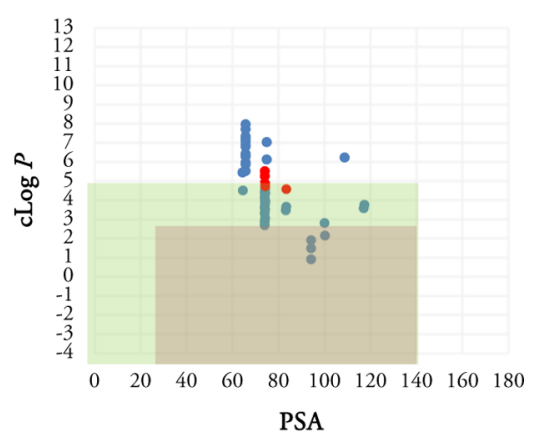

Publication 4

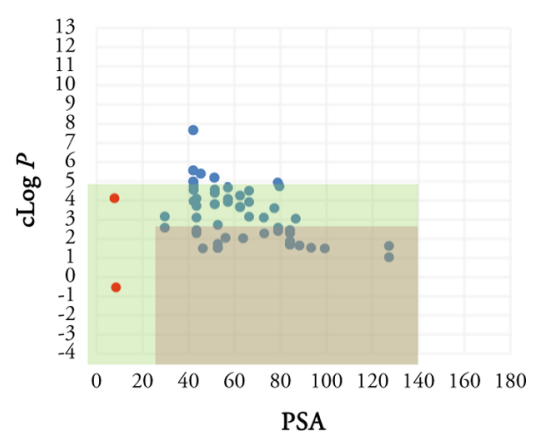

Figure 6. Scatterplots of the selected publications using $\operatorname{clog} P$ and PSA as two variables. Green rectangle determines the QED space and pink rectangle represents our putative TB space.

rising patterns sometimes were mostly inside or mostly/ completely outside the Lipinski and putative TB spaces (Figure $4 c, e)$.

The remaining publications present a more diverse pattern or no specific pattern such as publication 4 . Compounds 40-53 are the structures of the most active compounds (red dots) in the presented publications in Figure 4.

Since the scatterplots of the compounds using MW and $\operatorname{clog} P$ provided a great concept of the physiochemical properties, we decided to evaluate the scatterplots of TB drugs and the synthetic compounds using clog $P$ and PSA as they are the two physiochemical properties in which the most differences between TB drugs and the analyzed synthetic compounds are observed. Figure 5a shows the scatterplots of TB drugs using $\operatorname{clog} P$ and PSA as two variables. A QED space with $-5 \leq \operatorname{clog} P$ $\leq 5$ and $\mathrm{PSA} \leq 140 \AA$ and a putative TB space with $-4 \leq \operatorname{clog} P$ $\leq 3$ and $30 \leq \mathrm{PSA} \leq 140 \AA$ A were selected for further evaluation. The putative TB space is selected based on the appropriate ranges for $\operatorname{cog} P$ and PSA properties and the cluster of TB drugs observed in this region. About $70 \%$ of TB drugs are located in a QED space, and 56\% (22 drugs) are in the putative TB space. Five TB drugs which have PSA of more than $140 \AA$ are all naturederived TB drugs: streptomycin 5, amikacin 6, rifampicin 2, clarithromycin 19, and spectinamide 1810 24. Three marketed TB drugs (clofazimine 15, bedaquiline 16, and delamanid 17) and four TB drugs in clinical trials (TBAJ-587 25, TBAJ-876 26, TBI166 31, and telacebec Q203 39) are outside and above both QED and the putative TB spaces due to higher $\operatorname{cog} P$ values than 5. Pretomanid 20, BTZ043 27, macozinone 36, and SQ109 38 are four TB drugs located outside and above the putative TB space but still in the QED space, with $3 \leq \operatorname{clog} P \leq 5$.
Meropenem 18 is the only TB drug observed outside and below the putative TB space but still is in the QED space, with $\operatorname{cog} P$ of less than -4 .

The scatterplots of synthetic compounds showing in Figure 5 b reveal that $59 \%$ of synthetic compounds are in the QED space, while this percentage is only $18 \%$ in the putative TB space. The majority of violated compounds outside the QED space have higher $\operatorname{cog} P$ values, while only $3 \%$ of synthetic compounds show PSA of more than $140 \AA$. Figure $5 \mathrm{c}$ shows that about half of the most active synthetic compounds (51\%) are in the QED space and only $13 \%$ are located in the putative TB space. Similar to the scatterplots for all synthetic compounds, the majority of violated active compounds outside the QED space have higher clog $P$ values, and only one compound is out of the QED space due to a higher PSA value.

Scatterplots of the selected publications using clog $P$ and PSA as two variables are also depicted in Figure 6. The clusters observed in publications 5 and 28 in Figure 4 are replaced by a spread in the PSA dimension in those publications in Figure 6. This pattern is also observed in almost half of the analyzed publications due to synthesis of the series of similar analogues (Figure S3). The same $\operatorname{clog} P$ variation is also detected in publications 16 and 19 similar to Figure 4 with a further resolution in the PSA dimension. Similar to scatterplots of compounds using MW and $\operatorname{clog} P$, the remaining publications present a more diverse pattern or no specific pattern. In publication 4, compound 19 and one more compound have -4 $\leq \operatorname{clog} P \leq 3$; however, they have very low PSA values of less than 30 A. All reported synthetic compounds in publications 18 , 29 , and 30 are located in the QED space; however, no compound is observed in either QED or the putative TB space in 
Table 2. Categories of TB Drugs According to their Physiochemical Properties

\begin{tabular}{l|l|l|l}
$\begin{array}{l}\text { Drugs in the putative TB } \\
\text { space }\end{array}$ & Drugs with $\log P>5$ & First line drugs & Third line drugs \\
$\begin{array}{l}\text { Drugs with } \log P<-4 \\
\text { Drugs with } 3<\log P<5\end{array}$ & Drugs with PSA $>140 \AA$ & Second line drugs & Candidates in clinical trials \\
\hline
\end{tabular}

\begin{tabular}{|c|c|c|c|c|c|c|c|c|c|}
\hline No. & Name & MW & $\log P$ & HBD & HBA & PSA & ROTB & Ro5 & No. Violation \\
\hline 1 & Isoniazid & 137.14 & -0.69 & 2 & 3 & 68.01 & 1 & Pass & o \\
\hline 3 & Pyrazinamide & 123.11 & -1.23 & 1 & 3 & 68.87 & 1 & Pass & o \\
\hline 4 & Ethambutol & $204 \cdot 31$ & -0.06 & 4 & 4 & 64.52 & 9 & Pass & o \\
\hline 7 & p-amino salicylic acid & 153.14 & 0.83 & 3 & 4 & 83.55 & 1 & Pass & o \\
\hline 8 & Cycloserine & 102.09 & -2.42 & 2 & 3 & 64.35 & o & Pass & o \\
\hline 9 & Levofloxacin & 361.37 & 0.51 & 1 & 7 & $73 \cdot 32$ & 2 & Pass & o \\
\hline 10 & Moxifloxacin & 401.44 & -0.50 & 2 & 7 & 82.11 & 4 & Pass & o \\
\hline 11 & Ethionamide & 166.24 & 1.33 & 1 & 1 & 38.91 & 2 & Pass & o \\
\hline 12 & Prothionamide & 180.27 & 1.77 & 1 & 1 & 38.91 & 3 & Pass & o \\
\hline 13 & Terizidone & 302.29 & -0.34 & 2 & 6 & 101.38 & 4 & Pass & o \\
\hline 14 & Linezolid & 337.35 & 0.64 & 1 & 5 & 71.11 & 4 & Pass & o \\
\hline 21 & Sutezolid & 353.41 & 1.22 & 1 & 4 & 61.88 & 4 & Pass & o \\
\hline 22 & GSK-286 & 329.40 & 1.53 & 2 & 4 & 70.67 & 4 & Pass & o \\
\hline 23 & Sanfetrinem & 233.20 & -0.43 & o & 3 & 55.84 & 4 & Pass & o \\
\hline 28 & OPC -167832 & 456.85 & 2.85 & 3 & 5 & 82.03 & 4 & Pass & o \\
\hline 29 & $\mathrm{SPR}_{720}$ & 428.47 & 2.26 & 4 & 6 & 125.05 & 5 & Pass & o \\
\hline 30 & TBA-7371 & 355.40 & 1.19 & 2 & 6 & 102.16 & 6 & Pass & o \\
\hline 32 & TBI-223 & 365.36 & 0.93 & 1 & 5 & 80.34 & 5 & Pass & o \\
\hline 33 & Auranofin & 678.48 & -1.02 & o & 5 & 114.43 & 15 & Pass & 1 \\
\hline 34 & Delpazolid & 308.31 & 0.11 & 1 & 6 & 68.61 & 3 & Pass & o \\
\hline 35 & GSK $_{3036656}$ & 293.94 & 0.00 & 3 & 5 & 84.94 & 4 & Pass & o \\
\hline 37 & Nitazoxanide & 307.28 & 2.12 & 1 & 5 & 111.43 & 5 & Pass & o \\
\hline 18 & Meropenem & 383.46 & -4.35 & 6 & 3 & 110.18 & 5 & Pass & 2 \\
\hline 5 & Streptomycin & 581.58 & -7.06 & 12 & 19 & 336.43 & 9 & Fail & 3 \\
\hline 6 & Amikacin & 585.61 & -8.58 & 13 & 17 & 331.94 & 10 & Fail & 3 \\
\hline 20 & Pretomanid & 359.26 & 3.63 & o & 6 & 88.65 & 6 & Pass & o \\
\hline 27 & BTZo43 & 431.39 & 3.65 & o & 7 & 94.27 & 3 & Pass & o \\
\hline 36 & Macozinone & 456.48 & 4.53 & o & 6 & 79.05 & 5 & Pass & o \\
\hline 38 & SQ109 & 330.56 & 4.64 & 2 & 2 & 24.06 & 9 & Pass & o \\
\hline 15 & Clofazimine & 473.40 & $7 \cdot 30$ & 1 & 4 & 39.99 & 4 & Pass & 1 \\
\hline 16 & Bedaquiline & 555.52 & 7.13 & 1 & 4 & 45.59 & 8 & Fail & 2 \\
\hline 17 & Delamanid & 534.49 & 6.14 & o & 8 & 101.12 & 9 & Fail & 2 \\
\hline 25 & TBAJ-587 & 614.51 & 5.78 & 1 & 8 & 86.17 & 11 & Fail & 2 \\
\hline 26 & TBAJ-876 & 643.54 & 5.15 & 2 & 11 & 128.52 & 12 & Fail & 3 \\
\hline 31 & TBL166 & 589.62 & 7.03 & 1 & 8 & 80.57 & 8 & Fail & 2 \\
\hline 39 & Telacebec Q203 & 557.01 & 6.72 & 1 & 4 & 58.87 & 8 & Fail & 2 \\
\hline 2 & Rifampicin & 822.95 & 2.95 & 6 & 14 & 220.15 & 5 & Fail & 3 \\
\hline 5 & Streptomycin & 581.58 & -7.06 & 12 & 19 & 336.43 & 9 & Fail & 3 \\
\hline 6 & Amikacin & 585.61 & $-8.5^{8}$ & 13 & 17 & 331.94 & 10 & Fail & 3 \\
\hline 19 & Clarithromycin & 733.94 & 2.76 & 4 & 13 & 182.91 & 8 & Fail & 2 \\
\hline 24 & Spectinamide 1810 & 468.51 & -2.50 & 7 & 11 & 174.66 & 5 & Fail & 2 \\
\hline
\end{tabular}

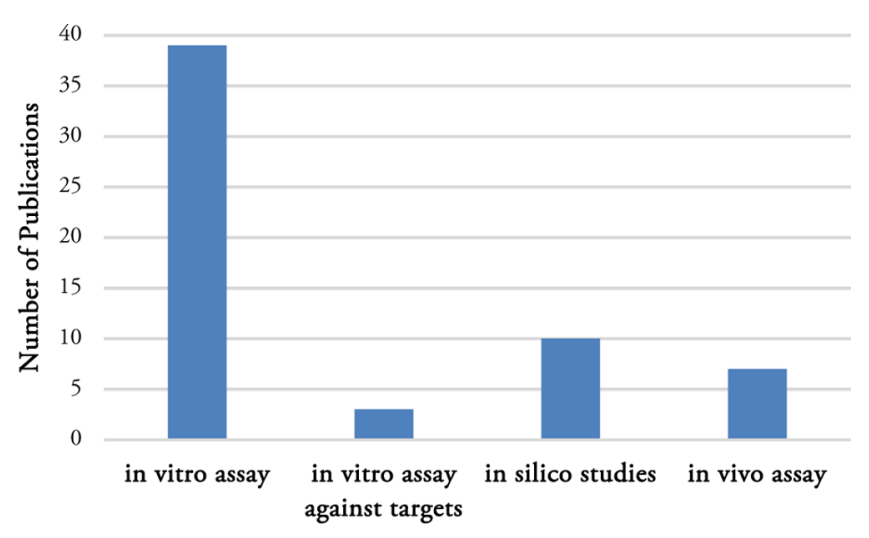

Figure 7. Analysis of the evaluation methods reported in the selected publications: in vitro assay, in vitro assay against targets, in silico studies, and in vivo assay.

publications 9, 22, and 27. In some publications such as publications 5 and 19, some compounds are in the QED space; however, no compound is detected in the putative TB space.
TB drugs categorized according to their physiochemical properties are shown in Table 2. Note that some drugs are repeated in different categories. The first 22 TB drugs are located in the putative TB space. Meropenem 18, streptomycin 5 , and amikacin 6 are three drugs which are administered intravenously with $\operatorname{cog} P<-4$. Four drugs have $3<\operatorname{clog} P<5$, and seven drugs have $\operatorname{clog} P>5$ including bedaquiline 16 and its derivatives, TBAJ-587 25 and TBAJ-876 26. Streptomycin 5, amikacin 6, rifampicin 2, clarithromycin 19, and spectinamide 181024 are five drugs with PSA > $140 \AA$.

The analyzed synthetic compounds in the reported publications were evaluated by in vitro and in vivo assays, in vitro assays against possible targets, and in silico studies (Figure 7). All publications used in vitro assays against M. tuberculosis, except one publication which did not report any evaluation of the synthetic compounds. Only three out of 40 publications performed in vitro assay against the possible target/enzyme. Molecular docking of all or selected synthetic compounds in a series against the possible target/enzyme was reported in 10 publications. Only 7 publications evaluated the most active compound in the series using in vivo assays. 


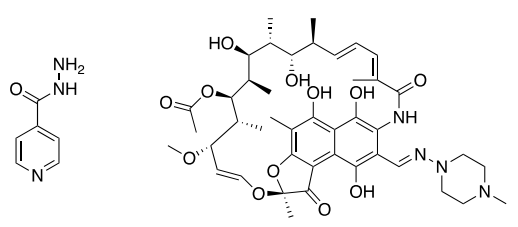

2

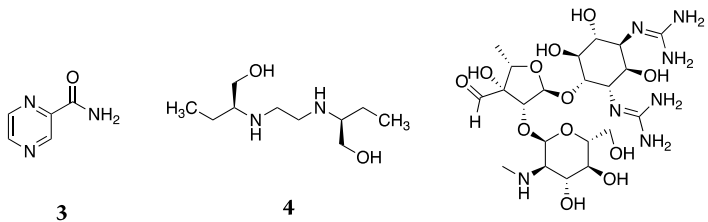

5

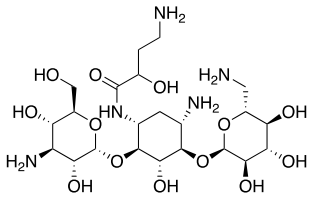

6<smiles>CC1COc2c(N3CCN(C)CC3)c(F)cc3c(=O)c(C(=O)O)cn(c23)OC1</smiles>

7

8

9

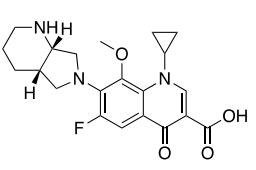

10<smiles>CCc1cc(C(N)=O)ccn1</smiles>

11<smiles>CCCc1cc(C(N)=S)ccn1</smiles>

12<smiles>O=C1NOCC1/N=C/c1ccc(/C=N/C2CONC2=O)cc1</smiles>

13<smiles>CC(=O)NCC1CN(c2ccc(N3CCOCC3)c(F)c2)C(=O)O1</smiles>

14

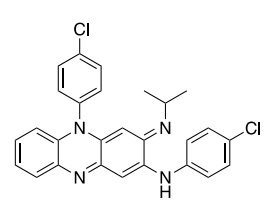

15

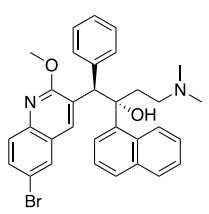

16

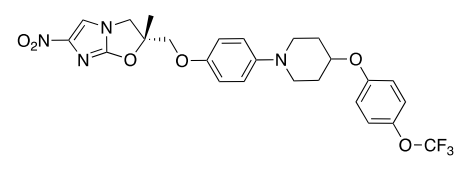

17

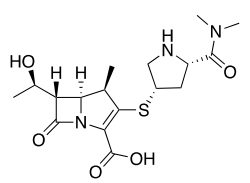

18

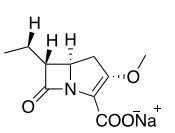

23

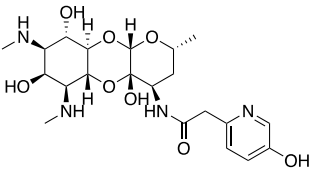

24<smiles>CCNC(=O)Nc1nc2cc(-c3cnc(C(C)(C)O)nc3)c(F)c(C3CCCO3)c2[nH]1</smiles>

29
25<smiles>COc1ncnc(Cn2cc(C(=O)NCCO)c3ncc(C)cc32)c1C</smiles>

30<smiles>COc1cc(C(c2cc3cc(Br)ccc3nc2OC)[C@@H](O)[C@H](O)CCN(C)C)cc(OC)c1OC</smiles>

26<smiles></smiles>

31<smiles>CC1COC2(CCN(c3nc(=O)c4cc(C(F)(F)F)cc([N+](=O)[O-])c4s3)CC2)O1</smiles>

27

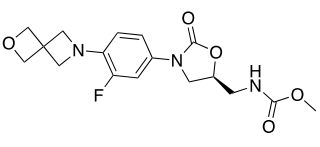

32

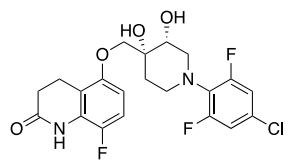

28

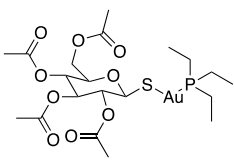

33

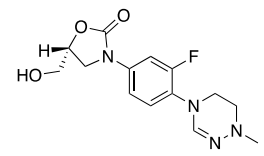

34<smiles>CCc1nc2ccc(Cl)cn2c1C(=O)NCc1ccc(N2CCC(c3ccc(OC(F)(F)F)cc3)CC2)cc1</smiles>

39
35<smiles>O=c1nc(N2CCN(CC3CCCCC3)CC2)sc2c([N+](=O)[O-])cc(C(F)(F)F)cc12</smiles>

36

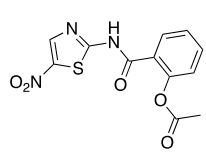

37

\section{CONCLUSIONS}

In this analysis, we evaluate 1271 synthetic anti-tubercular compounds using their physiochemical properties. In our analysis, we revealed that $\log P$ is a critical property which showed the most difference between TB drugs and the synthetic compounds. A huge shift to larger values for $\operatorname{clog} P$ was observed<smiles>CC(C)=CCC/C(C)=C/CNCCNC1C2CC3CC(C2)CC1C3</smiles>

38 among the synthetic compounds compared to TB drugs (Figure $2 \mathrm{~b}$ ). Selection of the second most apparent variation (Figure 2e) led to an analysis of clog $P$ against PSA. Combining the 3 parameters led us to propose $\operatorname{clog} P, \mathrm{MW}$, and PSA as the three important properties arising from this analysis. Clog $P-\mathrm{MW}$ (Figures 3 and 4 ) and clog P-PSA (Figures 5 and 6) provide the largest discrimination. Also, a new TB space with more 

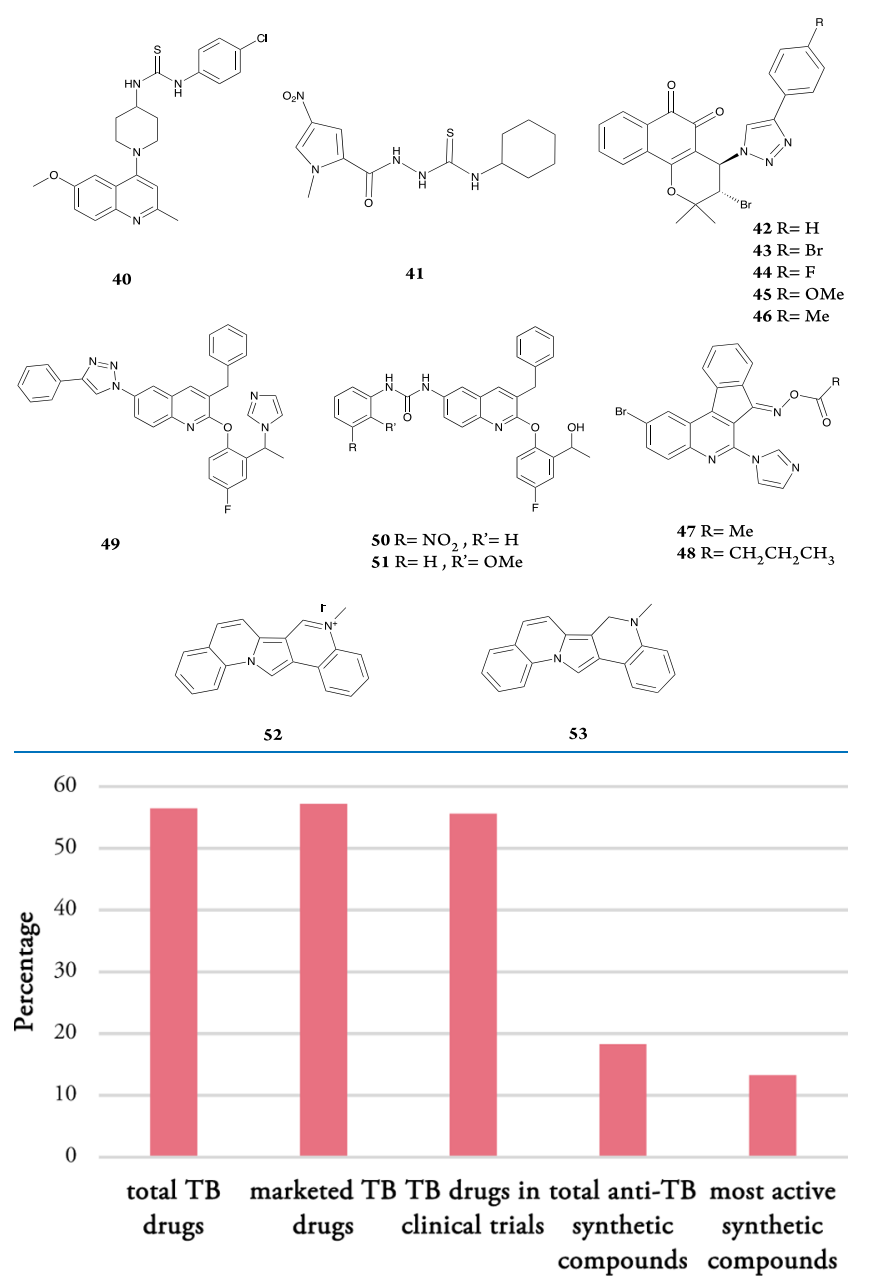

Figure 8. Percentage of total TB drugs, marketed TB drugs, TB drugs in clinical trials, total anti-TB synthetic compounds, and most active synthetic compounds in the proposed TB space.

appropriate values of MW $\leq 500,-4 \leq \log P \leq 3$ and $30 \leq$ PSA $\leq 140 \AA$ is proposed.

For example, bedaquiline 16, a second TB drug, is highly lipophilic and has a cardiac liability (prolongation of the QT interval) due to its potent inhibition of the cardiac potassium channel protein hERG. Therefore, synthesis of bedaquiline $\mathbf{1 6}$ analogues TBAJ-587 25 and TBAJ-876 26 was reported with lower lipophilicity, higher clearance, and lower risk for QT prolongation. ${ }^{55}$ The $\operatorname{clog} P$ value for bedaquiline 16 is 7.13 , and a significant decrease is observed in $\log P$ values of its derivatives TBAJ-587 25 and TBAJ-876 26 with 5.78 and 5.15 respectively; however, they may be further improved by modification to $\log P$ while retaining the PSA values.

The proposed TB space may be a useful and reliable guide to design new anti-mycobacterium compounds. Evaluation of TB drugs showed that about $56 \%$ of TB drugs are in the proposed TB space (Figure 8). Out of 39 TB drugs, 21 are marketed drugs and 18 are in clinical trials. Almost the same percentage of marketed TB drugs and those in clinical trials are observed in the proposed TB space that is 57 and $56 \%$, respectively. This number is significantly smaller in the anti-TB synthetic compounds. Only $18 \%$ of all synthetic compounds and $13 \%$ of the most active synthetic compounds are located in the proposed TB space.
The analysis of the evaluation methods reported in the selected publications also reveals that there is a lack of identification of the molecular target. Only a low percentage of the publications report targets for synthetic anti-mycobacterium compounds. This issue may be caused by some difficulties in identification of mode of action.

In conclusion, we have identified an area of the physicochemical space that is relatively underexplored in efforts to develop new TB drugs.

\section{ASSOCIATED CONTENT}

\section{Supporting Information}

The Supporting Information is available free of charge at https://pubs.acs.org/doi/10.1021/acsomega.0c03177.

Remaining histograms and scatterplots of the analyzed synthetic compounds (PDF)

Molecular formula strings (XLSX)

\section{AUTHOR INFORMATION}

\section{Corresponding Author}

Ronald J. Quinn - Griffith Institute for Drug Discovery, Griffith University, Nathan, Queensland 4111, Australia; $\odot$ orcid.org/ 0000-0002-4022-2623; Phone: (+61) 73735 6006;

Email: r.quinn@griffith.edu.au

\section{Author}

Sara Motamen - Griffith Institute for Drug Discovery, Griffith University, Nathan, Queensland 4111, Australia

Complete contact information is available at:

https://pubs.acs.org/10.1021/acsomega.0c03177

\section{Author Contributions}

The manuscript was written through contributions of all authors. All authors have given approval to the final version of the manuscript.

\section{Notes}

The authors declare no competing financial interest.

\section{ACKNOWLEDGMENTS}

This research was supported by the Bill and Melinda Gates Foundation (OPP1035218, OPP1174957). This research was supported by an Australian Research Council Discovery and Linkage Projects funding (DP160101429, LP120100485). S.M. acknowledges a Griffith University Postgraduate Research Scholarship and Griffith University International Postgraduate Research Scholarship.

\section{ABBREVIATIONS}

Mtb, Mycobacterium tuberculosis; TB, tuberculosis; QED, quantitative estimate of drug-likeness; MW, molecular weight; clog $P$, calculated $\log P$; HBD, hydrogen bond donor; HBA, hydrogen bond acceptor; PSA, polar surface area; ROTBs, rotatable bonds; $\mathrm{RNG}$, aromatic ring count

\section{REFERENCES}

(1) Global Tuberculosis Report 2019; World Health Organization: Geneva, 2019.

(2) Lipinski, C. A.; Lombardo, F.; Dominy, B. W.; Feeney, P. J. Experimental and computational approaches to estimate solubility and permeability in drug discovery and development settings. Adv. Drug Delivery Rev. 1997, 23, 3-25. 
(3) Lipinski, C. A. Lead-and drug-like compounds: the rule-of-five revolution. Drug Discovery Today: Technol. 2004, 1, 337-341.

(4) Bickerton, G. R.; Paolini, G. V.; Besnard, J.; Muresan, S.; Hopkins, A. L. Quantifying the chemical beauty of drugs. Nat. Chem. 2012, 4, 90. (5) Andrews, P. R.; Craik, D. J.; Martin, J. L. Functional group contributions to drug-receptor interactions. J. Med. Chem. 1984, 27, $1648-1657$.

(6) Klebe, G.; Böhm, H.-J. Energetic and entropic factors determining binding affinity in protein-ligand complexes. J. Recept. Signal Transduction 1997, 17, 459-473.

(7) Veber, D. F.; Johnson, S. R.; Cheng, H.-Y.; Smith, B. R.; Ward, K. W.; Kopple, K. D. Molecular properties that influence the oral bioavailability of drug candidates. J. Med. Chem. 2002, 45, 2615-2623.

(8) Teague, S. J.; Davis, A. M.; Leeson, P. D.; Oprea, T. The design of leadlike combinatorial libraries. Angew. Chem., Int. Ed. 1999, 38, 37433748.

(9) Nayak, N.; Ramprasad, J.; Dalimba, U. Synthesis and antitubercular and antibacterial activity of some active fluorine containing quinoline-pyrazole hybrid derivatives. J. Fluorine Chem. 2016, 183, 59-68.

(10) Anand, A.; Naik, R. J.; Revankar, H. M.; Kulkarni, M. V.; Dixit, S. R.; Joshi, S. D. A click chemistry approach for the synthesis of mono and bis aryloxy linked coumarinyl triazoles as anti-tubercular agents. Eur. J. Med. Chem. 2015, 105, 194-207.

(11) Balamurugan, K.; Jeyachandran, V.; Perumal, S.; Manjashetty, T. H.; Yogeeswari, P.; Sriram, D. A microwave-assisted, facile, regioselective Friedländer synthesis and antitubercular evaluation of 2, 9-diaryl-2, 3-dihydrothieno-[3, 2-b] quinolines. Eur. J. Med. Chem. 2010, 45, 682-688.

(12) Ganihigama, D. U.; Sureram, S.; Sangher, S.; Hongmanee, P.; Aree, T.; Mahidol, C.; Ruchirawat, S.; Kittakoop, P. Antimycobacterial activity of natural products and synthetic agents: Pyrrolodiquinolines and vermelhotin as anti-tubercular leads against clinical multidrug resistant isolates of Mycobacterium tuberculosis. Eur. J. Med. Chem. 2015, 89, 1-12.

(13) Medapi, B.; Renuka, J.; Saxena, S.; Sridevi, J. P.; Medishetti, R.; Kulkarni, P.; Yogeeswari, P.; Sriram, D. Design and synthesis of novel quinoline-aminopiperidine hybrid analogues as Mycobacterium tuberculosis DNA gyraseB inhibitors. Bioorg. Med. Chem. 2015, 23, 2062-2078.

(14) Eswaran, S.; Adhikari, A. V.; Pal, N. K.; Chowdhury, I. H. Design and synthesis of some new quinoline-3-carbohydrazone derivatives as potential antimycobacterial agents. Bioorg. Med. Chem. Lett. 2010, 20, $1040-1044$

(15) Muscia, G. C.; Buldain, G. Y.; Asís, S. E. Design, synthesis and evaluation of acridine and fused-quinoline derivatives as potential antituberculosis agents. Eur. J. Med. Chem. 2014, 73, 243-249.

(16) Tanwar, B.; Kumar, A.; Yogeeswari, P.; Sriram, D.; Chakraborti, A. K. Design, development of new synthetic methodology, and biological evaluation of substituted quinolines as new anti-tubercular leads. Bioorg. Med. Chem. Lett. 2016, 26, 5960-5966.

(17) Upadhayaya, R. S.; Kulkarni, G. M.; Vasireddy, N. R.; Vandavasi, J. K.; Dixit, S. S.; Sharma, V.; Chattopadhyaya, J. Design, synthesis and biological evaluation of novel triazole, urea and thiourea derivatives of quinoline against Mycobacterium tuberculosis. Bioorg. Med. Chem. 2009, 17, 4681-4692.

(18) Dixit, P. P.; Dixit, P. P.; Thore, S. N. Hybrid triazoles: Design and synthesis as potential dual inhibitor of growth and efflux inhibition in tuberculosis. Eur. J. Med. Chem. 2016, 107, 38-47.

(19) Jain, P. P.; Degani, M. S.; Raju, A.; Anantram, A.; Seervi, M.; Sathaye, S.; Ray, M.; Rajan, M. G. R. Identification of a novel class of quinoline-oxadiazole hybrids as anti-tuberculosis agents. Bioorg. Med. Chem. Lett. 2016, 26, 645-649.

(20) Yang, C.-L.; Tseng, C.-H.; Chen, Y.-L.; Lu, C.-M.; Kao, C.-L.; Wu, M.-H.; Tzeng, C.-C. Identification of benzofuro [2, 3-b] quinoline derivatives as a new class of antituberculosis agents. Eur. J. Med. Chem. 2010, 45, 602-607.
(21) Eswaran, S.; Adhikari, A. V.; Ajay Kumar, R. New 1, 3-oxazolo [4, 5-c] quinoline derivatives: Synthesis and evaluation of antibacterial and antituberculosis properties. Eur. J. Med. Chem. 2010, 45, 957-966.

(22) Anguru, M. R.; Taduri, A. K.; Bhoomireddy, R. D.; Jojula, M.; Gunda, S. K. Novel drug targets for Mycobacterium tuberculosis: 2heterostyrylbenzimidazoles as inhibitors of cell wall protein synthesis. Chem. Cent. J. 2017, 11, 68.

(23) Upadhayaya, R. S.; Vandavasi, J. K.; Kardile, R. A.; Lahore, S. V.; Dixit, S. S.; Deokar, H. S.; Shinde, P. D.; Sarmah, M. P.; Chattopadhyaya, J. Novel quinoline and naphthalene derivatives as potent antimycobacterial agents. Eur. J. Med. Chem. 2010, 45, 18541867.

(24) Jardim, G. A. M.; Cruz, E. H.; Valença, W. O.; Resende, J. M.; Rodrigues, B. L.; Ramos, D. F.; Oliveira, R. N.; Silva, P. E.; Silva Júnior, E. N. d. On the search for potential antimycobacterial drugs: synthesis of naphthoquinoidal, phenazinic and 1, 2, 3-triazolic compounds and evaluation against Mycobacterium tuberculosis. J. Braz. Chem. Soc. 2015, 26, 1013-1027.

(25) Jain, P. P.; Degani, M. S.; Raju, A.; Ray, M.; Rajan, M. G. R. Rational drug design based synthesis of novel arylquinolines as antituberculosis agents. Bioorg. Med. Chem. Lett. 2013, 23, 6097-6105.

(26) Cappoen, D.; Jacobs, J.; Nguyen Van, T.; Claessens, S.; Diels, G.; Anthonissen, R.; Einarsdottir, T.; Fauville, M.; Verschaeve, L.; Huygen, K.; De Kimpe, N. Straightforward palladium-mediated synthesis and biological evaluation of benzo [j] phenanthridine-7, 12-diones as antituberculosis agents. Eur. J. Med. Chem. 2012, 48, 57-68.

(27) Upadhayaya, R. S.; Shinde, P. D.; Kadam, S. A.; Bawane, A. N.; Sayyed, A. Y.; Kardile, R. A.; Gitay, P. N.; Lahore, S. V.; Dixit, S. S.; Földesi, A.; Chattopadhyaya, J. Synthesis and antimycobacterial activity of prodrugs of indeno [2, 1-c] quinoline derivatives. Eur. J. Med. Chem. 2011, 46, 1306-1324.

(28) Senthilkumar, P.; Dinakaran, M.; Banerjee, D.; Devakaram, R. V.; Yogeeswari, P.; China, A.; Nagaraja, V.; Sriram, D. Synthesis and antimycobacterial evaluation of newer 1-cyclopropyl-1, 4-dihydro-6fluoro-7-(substituted secondary amino)-8-methoxy-5-(sub)-4-oxoquinoline-3-carboxylic acids. Bioorg. Med. Chem. 2008, 16, 2558-2569.

(29) Choi, P. J.; Sutherland, H. S.; Tong, A. S. T.; Blaser, A.; Franzblau, S. G.; Cooper, C. B.; Lotlikar, M. U.; Upton, A. M.; Guillemont, J.; Motte, M.; Queguiner, L.; Andries, K.; Van den Broeck, W.; Denny, W. A.; Palmer, B. D. Synthesis and evaluation of analogues of the tuberculosis drug bedaquiline containing heterocyclic B-ring units. Bioorg. Med. Chem. Lett. 2017, 27, 5190-5196.

(30) Kathrotiya, H. G.; Patel, M. P. Synthesis and identification of $\beta$ aryloxyquinoline based diversely fluorine substituted $\mathrm{N}$-aryl quinolone derivatives as a new class of antimicrobial, antituberculosis and antioxidant agents. Eur. J. Med. Chem. 2013, 63, 675-684.

(31) Govender, T.; Kruger, H. G.; Makatini, M.; Onajole, O. K. Synthesis and NMR elucidation of pentacyclo-undecane diamine derivatives as potential anti-tuberculosis drugs. Struct. Chem. 2008, 19, 719.

(32) Scalacci, N.; Brown, A. K.; Pavan, F. R.; Ribeiro, C. M.; Manetti, F.; Bhakta, S.; Maitra, A.; Smith, D. L.; Petricci, E.; Castagnolo, D. Synthesis and SAR evaluation of novel thioridazine derivatives active against drug-resistant tuberculosis. Eur. J. Med. Chem. 2017, 127, 147158.

(33) Palmer, B. D.; Thompson, A. M.; Sutherland, H. S.; Blaser, A.; Kmentova, I.; Franzblau, S. G.; Wan, B.; Wang, Y.; Ma, Z.; Denny, W. A. Synthesis and Structure- Activity Studies of Biphenyl Analogues of the Tuberculosis Drug (6 S)-2-Nitro-6-\{[4-(trifluoromethoxy) benzyl] oxy\}-6, 7-dihydro-5 H-imidazo [2, 1-b][1,3] oxazine (PA-824). J. Med. Chem. 2010, 53, 282-294.

(34) Kalalbandi, V. K. A.; Seetharamappa, J.; Katrahalli, U.; Bhat, K. G. Synthesis, crystal studies, anti-tuberculosis and cytotoxic studies of 1[(2E)-3-phenylprop-2-enoyl]-1H-benzimidazole derivatives. Eur. J. Med. Chem. 2014, 79, 194-202.

(35) Kachhadia, V. V.; Patel, M.; Joshi, H. Synthesis of isoxazoles and cyanopyridines bearing benzo (b) thiophene nucleus as potential antitubercular and antimicrobial agents. J. Sci., Islamic Repub. Iran 2004, $15,47-51$. 
(36) Rane, R. A.; Naphade, S. S.; Bangalore, P. K.; Palkar, M. B.; Shaikh, M. S.; Karpoormath, R. Synthesis of novel 4-nitropyrrole-based semicarbazide and thiosemicarbazide hybrids with antimicrobial and anti-tubercular activity. Bioorg. Med. Chem. Lett. 2014, 24, 3079-3083.

(37) Costa, M. S.; Boechat, N.; Rangel, É. A.; Da Silva, F. d. C.; De Souza, A. M. T.; Rodrigues, C. R.; Castro, H. C.; Junior, I. N.; Lourenço, M. C. S.; Wardell, S. M. S. V.; Ferreira, V. F. Synthesis, tuberculosis inhibitory activity, and SAR study of N-substituted-phenyl-1, 2, 3triazole derivatives. Bioorg. Med. Chem. 2006, 14, 8644-8653.

(38) Manvar, A.; Malde, A.; Verma, J.; Virsodia, V.; Mishra, A.; Upadhyay, K.; Acharya, H.; Coutinho, E.; Shah, A. Synthesis, antitubercular activity and 3D-QSAR study of coumarin-4-acetic acid benzylidene hydrazides. Eur. J. Med. Chem. 2008, 43, 2395-2403.

(39) Nayyar, A.; Patel, S. R.; Shaikh, M.; Coutinho, E.; Jain, R. Synthesis, anti-tuberculosis activity and 3D-QSAR study of amino acid conjugates of 4-(adamantan-1-yl) group containing quinolines. Eur. J. Med. Chem. 2009, 44, 2017-2029.

(40) Patel, S. R.; Gangwal, R.; Sangamwar, A. T.; Jain, R. Synthesis, biological evaluation and 3D QSAR study of 2, 4-disubstituted quinolines as anti-tuberculosis agents. Eur. J. Med. Chem. 2015, 93, $511-522$.

(41) Patel, S. R.; Gangwal, R.; Sangamwar, A. T.; Jain, R. Synthesis, biological evaluation and 3D-QSAR study of hydrazide, semicarbazide and thiosemicarbazide derivatives of 4-(adamantan-1-yl) quinoline as anti-tuberculosis agents. Eur. J. Med. Chem. 2014, 85, 255-267.

(42) Guzman, J. D.; Pesnot, T.; Barrera, D. A.; Davies, H. M.; McMahon, E.; Evangelopoulos, D.; Mortazavi, P. N.; Munshi, T.; Maitra, A.; Lamming, E. D.; Angell, R.; Gershater, M. C.; Redmond, J. M.; Needham, D.; Ward, J. M.; Cuca, L. E.; Hailes, H. C.; Bhakta, S. Tetrahydroisoquinolines affect the whole-cell phenotype of Mycobacterium tuberculosis by inhibiting the ATP-dependent MurE ligase. J. Antimicrob. Chemother. 2015, 70, 1691-1703.

(43) Alsayed, S. S. R.; Lun, S.; Luna, G.; Beh, C. C.; Payne, A. D.; Foster, N.; Bishai, W. R.; Gunosewoyo, H. Design, synthesis, and biological evaluation of novel arylcarboxamide derivatives as antitubercular agents. RSC Adv. 2020, 10, 7523-7540.

(44) Suthagar, K.; Jiao, W.; Munier-Lehmann, H.; Fairbanks, A. J. Synthesis of sulfamide analogues of deoxthymidine monophosphate as potential inhibitors of mycobacterial cell wall biosynthesis. Carbohydr. Res. 2018, 457, 32-40.

(45) Hearn, M. J.; Cynamon, M. H.; Chen, M. F.; Coppins, R.; Davis, J.; Joo-On Kang, H.; Noble, A.; Tu-Sekine, B.; Terrot, M. S.; Trombino, D. Preparation and antitubercular activities in vitro and in vivo of novel Schiff bases of isoniazid. Eur. J. Med. Chem. 2009, 44, 4169-4178.

(46) Marvadi, S. k.; Krishna, V. S.; Surineni, G.; Srilakshmi Reshma, R.; Sridhar, B.; Sriram, D.; Kantevari, S. Synthesis, in vitro, and in vivo (Zebra fish) antitubercular activity of 7, 8-dihydroquinolin-5 (6H)ylidenehydrazinecarbothioamides. Bioorg. Chem. 2020, 96, 103626.

(47) Guardia, A.; Baiget, J.; Cacho, M.; Pérez, A.; Ortega-Guerra, M.; Nxumalo, W.; Khanye, S. D.; Rullas, J.; Ortega, F.; Jiménez, E.; PérezHerrán, E.; Fraile-Gabaldón, M. T.; Esquivias, J.; Fernández, R.; PorrasDe Francisco, E.; Encinas, L.; Alonso, M.; Giordano, I.; Rivero, C.; Miguel-Siles, J.; Osende, J. G.; Badiola, K. A.; Rutledge, P. J.; Todd, M. H.; Remuiñán, M.; Alemparte, C. Easy-To-Synthesize Spirocyclic Compounds Possess Remarkable in Vivo Activity against Mycobacterium tuberculosis. J. Med. Chem. 2018, 61, 11327-11340.

(48) Cheng, Y.-J.; Liu, Z.-Y.; Liang, H.-J.; Fang, C.-T.; Zhang, N.-N.; Zhang, T.-Y.; Yan, M. Discovery of (3-Benzyl-5-hydroxyphenyl) carbamates as new antitubercular agents with potent in vitro and in vivo efficacy. Molecules 2019, 24, 2021.

(49) Instant JChem, ChemAxon. https://chemaxon.com/products/ instant-jchem (accessed March 1, 2020).

(50) The Working Group for New TB Drugs Home Page. https:// www.newtbdrugs.org/pipeline/clinical (accessed April 25, 2020).

(51) Dashti, Y.; Grkovic, T.; Quinn, R. J. Predicting natural product value, an exploration of anti-TB drug space. Nat. Prod. Rep. 2014, 31, 990-998.
(52) O'Shea, R.; Moser, H. E. Physicochemical properties of antibacterial compounds: implications for drug discovery. J. Med. Chem. 2008, 51, 2871-2878.

(53) Brown, D. G.; May-Dracka, T. L.; Gagnon, M. M.; Tommasi, R. Trends and exceptions of physical properties on antibacterial activity for Gram-positive and Gram-negative pathogens. J. Med. Chem. 2014, 57, 10144-10161.

(54) Pascolutti, M.; Quinn, R. J. Natural products as lead structures: chemical transformations to create lead-like libraries. Drug Discovery Today 2014, 19, 215-221.

(55) Sutherland, H. S.; Tong, A. S.; Choi, P. J.; Blaser, A.; Conole, D.; Franzblau, S. G.; Lotlikar, M. U.; Cooper, C. B.; Upton, A. M.; Denny, W. A. 3, 5-Dialkoxypyridine analogues of bedaquiline are potent antituberculosis agents with minimal inhibition of the hERG channel. Bioorg. Med. Chem. 2019, 27, 1292-1307. 